\title{
Current-voltage relation for thin tunnel barriers: Parabolic barrier model
}

\author{
Hansen, Kim; Brandbyge, Mads
}

Published in:

Journal of Applied Physics

Link to article, DOI:

$10.1063 / 1.1650896$

Publication date:

2004

Document Version

Publisher's PDF, also known as Version of record

Link back to DTU Orbit

Citation (APA):

Hansen, K., \& Brandbyge, M. (2004). Current-voltage relation for thin tunnel barriers: Parabolic barrier model. Journal of Applied Physics, 95(7), 3582-3586. https://doi.org/10.1063/1.1650896

\section{General rights}

Copyright and moral rights for the publications made accessible in the public portal are retained by the authors and/or other copyright owners and it is a condition of accessing publications that users recognise and abide by the legal requirements associated with these rights.

- Users may download and print one copy of any publication from the public portal for the purpose of private study or research.

- You may not further distribute the material or use it for any profit-making activity or commercial gain

- You may freely distribute the URL identifying the publication in the public portal

If you believe that this document breaches copyright please contact us providing details, and we will remove access to the work immediately and investigate your claim 


\title{
Current-voltage relation for thin tunnel barriers: Parabolic barrier model
}

\author{
Kim Hansen \\ CAMP and Institute of Physics and Astronomy, University of Aarhus, DK 8000 Aarhus C, Denmark \\ Mads Brandbyge ${ }^{\text {a) }}$ \\ MIC, Department of Micro and Nanotechnology, Technical University of Denmark, Bldg. 345E, \\ DK-2800 Kongens Lyngby, Denmark
}

(Received 22 August 2003; accepted 5 January 2004)

\begin{abstract}
We derive a simple analytic result for the current-voltage curve for tunneling of electrons through a thin uniform insulating layer modeled by a parabolic barrier. Our model, which goes beyond the Wentzel-Kramers-Brillouin approximation, is applicable also in the limit of highly transparant barriers subject to high voltages, and thus provides a more realistic description for this situation compared to the widely used rectangular barrier model. (C) 2004 American Institute of Physics.
\end{abstract}

[DOI: $10.1063 / 1.1650896]$

\section{INTRODUCTION}

Tunneling in metal-vacuum-metal or metal-insulatormetal contacts is a very old and well-studied subject. ${ }^{1-3}$ Various formulas for the current-voltage characteristics were derived in a series of classical papers in the early 1960's for free electron electrodes. ${ }^{4-6}$ These treatments were mainly intended for calculating the current-voltage curves through fairly thick insulating layers compared to the de Broglie wavelength of the electrons at the Fermi energy $\left(\lambda_{F}\right)$. In this case the Wentzel-Kramers-Brillouin (WKB) approximation $^{7}$ is valid for the tunnel transmission and widely used to obtain simple analytic expressions for the current-voltage curves for simple barrier models which, in the end, are easy to apply in the analysis of experimental current-voltage curves. The WKB approximation is quite accurate when dealing with opaque barriers, but it is not applicable to barriers with high transparency: For thin and low barriers (relative to the Fermi energy, $E_{F}$ ) the WKB approximation breaks down. This becomes especially important for large applied voltages which can lower the tunnel barrier substantially. Thus it is interesting to consider an alternative analytic model avoiding the WKB and still tractable when fitting to experimental data. Our motivation for deriving an analytic expression for the current-voltage characteristic for a simple thin-barrier model stems from a study of atomicsized gold contacts in a scanning-tunneling microscope setup $^{8}$ where nonlinear current-voltage curves were related to tunneling through a thin layer of contaminants in the contact.

In the next section the general formulas for the current are discussed. Then the truncated parabolic barrier model is introduced followed by the derivation of the current-voltage characteristics for this model and illustration of the model by an example.

\section{EXPRESSIONS FOR THE CURRENT}

Often details about the contact geometry and tunneling region are unknown and the experiments are discussed in

\footnotetext{
${ }^{a)}$ Electronic mail: mbr@mic.dtu.dk
}

terms of the simple barrier geometry shown in Fig. 1(a). Here we consider tunneling through an area $A=L_{x} L_{y}$ through a barrier $\phi(z)$ of thickness $d$.

We assume that the electrons do not lose energy in inelastic scattering inside the barrier. In this case the current $I$ for voltage $V$ is given by the Landauer-Büttiker formula ${ }^{9,10}$

$$
\begin{aligned}
I(V)= & \frac{2 e}{h} \int_{0}^{\infty} d E[f(E)-f(E+\mathrm{eV})] \sum_{\mathbf{k}_{\perp}} T_{\mathbf{k}_{\perp}}(E, V) \\
= & \frac{A e}{2 \pi^{2} h} \int_{0}^{\infty} d E[f(E)-f(E \\
& +\mathrm{eV})] \int d \mathbf{k}_{\perp} T_{\mathbf{k}_{\perp}}(E, V),
\end{aligned}
$$

where $f(E)$ is the Fermi-Dirac distribution, $\mathbf{k}_{\perp}$, is the wave vector perpendicular to the $z$-axis, and $T_{\mathbf{k}_{\perp}}(E, V)$ is the tunnel probability for an incoming electron with $\mathbf{k}_{\perp}=\left(k_{x}, k_{y}\right)$ and total energy $E$. We asume that the contact dimensions $L_{x}, L_{y}$ are much larger $\lambda_{F}$ so the quantization in the transverse direction can be neglected and $\mathbf{k}_{\perp}$ is continous. The integration over $\mathbf{k}_{\perp}$ is restricted to values which conserve $E$ and $\mathbf{k}_{\perp}$. For free electron dispersions the transmission probability depends only on the kinetic energy $E_{z}$ along $z$ ( $E$ $=E_{\perp}+E_{z}$ ), and we may write

$$
T_{\mathbf{k}_{\perp}}(E)=T_{1 \mathrm{D}}\left(E_{z}\right)=T_{1 \mathrm{D}}\left(E-E_{\perp}\right) .
$$

$T_{1 \mathrm{D}}\left(E_{z}, V\right)$ is simply the probability for tunneling through the one-dimensional (1D) potential $\phi(V ; z)$.

We find it instructive to define the mean transmission $T_{3 \mathrm{D}}$ for an incoming electron with energy $E$ by averaging over all possible values of $E_{z}$

$$
T_{3 \mathrm{D}}(E, V) \equiv \frac{1}{E} \int_{0}^{E} T_{1 \mathrm{D}}\left(E_{z}, V\right) d E_{z}
$$

whereby the current is written as

$$
I(V)=A \frac{4 \pi m e}{h^{3}} \int_{0}^{\infty} d E[f(E)-f(E+\mathrm{eV})] E T_{3 \mathrm{D}}(E, V) .
$$



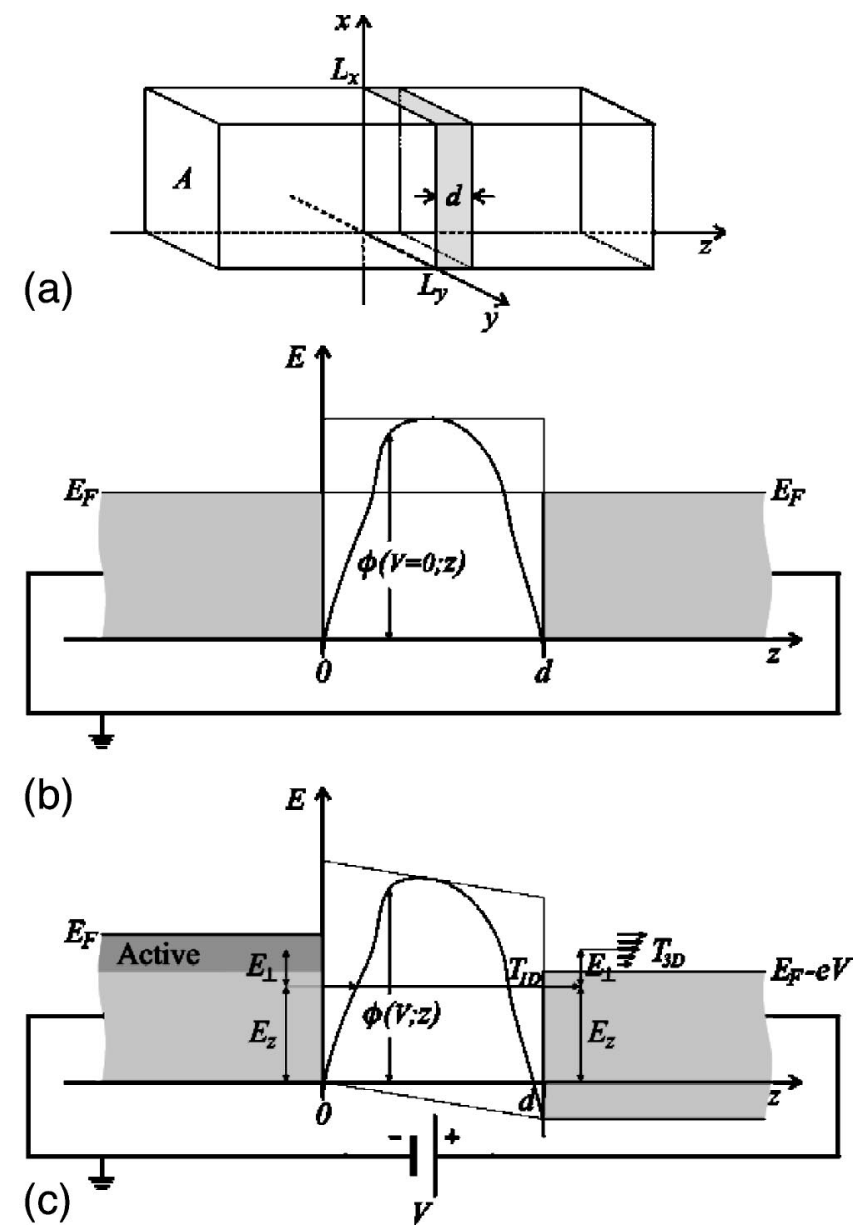

FIG. 1. (a) Model geometry used for calculating the voltage dependent tunnel current through a thin insulating film. (b) Energy diagram for tunneling through a thin barrier with no bias voltage applied. (c) Energy diagram for a applied positive voltage $V$. The thin dotted lines illustrate how the generated electric field in the film deforms the zero voltage barrier assuming no charge redistribution in the barrier. Only electrons in the energy window defined by the Fermi functions $f(E)-f(E+\mathrm{eV})$ contribute to the current.

$T_{3 \mathrm{D}}$ increases with increasing energy since here the electrons with $E_{z}$ close to $E$ penetrate a smaller potential barrier. This is illustrated by the arrows in Fig. 1(b). $T_{3 \mathrm{D}}$ will also increase with increasing $V$. We note that for a transparent barrier $\left(T_{3 \mathrm{D}}=1\right)$ and in limit of small voltages $\left(\mathrm{eV} \ll E_{F}\right)$ Eq. (4) yields

$$
I(V)=\pi A \frac{2 E_{F} m}{h^{2}} \frac{2_{e}^{2}}{h} V=\frac{\pi A}{\lambda_{F}^{2}} G_{0} V=G_{S} V,
$$

where $G_{0}=2_{e}^{2} / h \sim 1 / 12.9 \mathrm{k} \Omega$ is the quantum unit of conductance and $G_{S}$ is known as the Sharvin conductance. ${ }^{11}$ It is natural to rewrite the expression for the current such that $G_{S}$ is used as a prefactor

$$
I(V)=G_{S} \mathcal{T}(V) V,
$$

where

$$
\mathcal{T}(V)=\frac{\int_{0}^{\infty} d E[f(E)-f(E+\mathrm{eV})] E T_{3 \mathrm{D}}(E, V)}{\mathrm{eV} E_{F}}
$$

is the mean transmission probability averaged over all electrons in the energy window $\mathrm{eV}$ below the Fermi energy in the

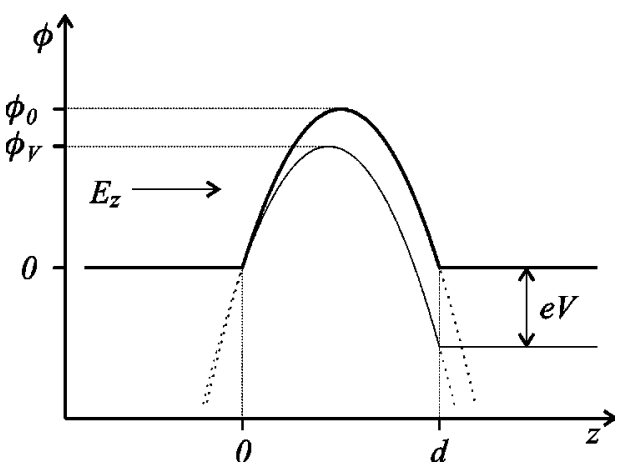

FIG. 2. Solid lines: The truncated parabola model without (thick line) and with (thin line) an applied bias voltage. The dotted lines show the continuations of the parabolas.

left electrode. By writing the current in the form of Eq. (6) we see that the interesting physics such as nonlinearities is contained in the voltage dependence of $\mathcal{T}(V)$.

Since $T_{3 \mathrm{D}}(E, V)$ is most often a smoothly varying function of $E$ (on the scale of $k_{B} T$ ), we can use the Sommerfeld expansion $^{12}$ and write Eq. (7) as

$$
\mathcal{T}(V) \sim \mathcal{T}_{0}(V)+\Delta \mathcal{T}(V)
$$

where

$$
\mathcal{T}_{0}(V)=\frac{1}{\mathrm{eV} E_{F}} \int_{E_{F}-\mathrm{eV}}^{E_{F}} d E E T_{3 \mathrm{D}}(E, V)
$$

is the zero temperature mean transmission, and

$$
\Delta \mathcal{T}(V)=\frac{\pi^{2}}{6} \frac{\left(k_{B} T\right)^{2}}{\mathrm{eVE} E_{F}}\left[T_{1 \mathrm{D}}\left(E_{F}, V\right)-T_{1 \mathrm{D}}\left(E_{F}-\mathrm{eV}, V\right)\right]
$$

is the second order temperature correction to the mean transmission. In the following, we employ these formulas for a simple parabolic model potential.

\section{PARABOLIC BARRIER MODEL}

Simple square potential models and the WKB approximation $^{5}$ are often used in the analysis of experiments where little is known about the tunnel region, so a minimum of parameters are used for its description such as the barrier thickness and height. This has, e.g., been done for tunneling through organic monolayers. ${ }^{13,14}$ Here we focus on a barrier model which is again only described by a barrier height and thickness but is adequate for thin and low barriers, where the WKB approximation can not be applied. Our choice is the parabolic barrier, which, unlike the rectangular barrier is continuous at the electrodes, thereby removing the infinite forces at the surface and causing cusps in the $I-V$ curves. In our model, we place the parabolic barrier with height $\phi_{0}$ in the middle of the gap between the metal electrodes as shown by the thick solid line in Fig. 2 and write the barrier for $V$ $=0$

$$
\phi(0 ; z)= \begin{cases}0 & \text { if } z<0 \text { or } z>d, \\ \phi_{0}-\frac{1}{2} K\left(z-\frac{d}{2}\right)^{2} & \text { if } 0 \leqslant z \leqslant d .\end{cases}
$$


We select the curvature $K$ in Eq. (11) such that the barrier connects continuously to the bottom of the electrode potentials at the surfaces $[\phi(0 ; 0)=\phi(0 ; d)=0]$, whereby the shape of the barrier is fully described by $\phi_{0}$ and $d$

$$
K=\frac{8 \phi_{0}}{d^{2}} .
$$

We will neglect charge rearrangement inside the barrier, so the zero voltage barrier $\phi(V=0 ; z)$ is modified by $-\mathrm{eV} z / d$ when a bias voltage $V$ is applied ${ }^{1-5,15-17}$

$$
\phi(V ; z)=\phi(0 ; z)-\mathrm{eV} \frac{z}{d} .
$$

We can write the barrier at finite bias (shown as a thin solid line in Fig. 2) by using Eq. (13)

$$
\phi(V ; z)=\phi_{V}-\frac{4 \phi_{0}}{d^{2}}\left(z-z_{\max }\right)^{2} .
$$

The voltage-dependent barrier maximum located at $z_{\max }$, is given by

$$
\phi_{V}=\max [\phi(V ; z)]=\phi_{0}\left(1-\frac{1}{4} \frac{\mathrm{eV}}{\phi_{0}}\right)^{2} .
$$

The presence of an electric field in the film lowers the barrier height by $\mathrm{eV} / 2$ to lowest order in $\mathrm{eV}$. Unlike rectangular and image barriers, the parabolic barrier does not change its shape (curvature) when a voltage is applied. Only the barrier height and the maximum position change. In the following, we will see that this is a very convenient feature of the model.

The WKB tranmission is evaluated to the simple expression

$$
T_{1 \mathrm{D}}^{\mathrm{WKB}}\left(E_{z}, V\right)=\exp \left[-\gamma\left(\phi_{V}-E_{z}\right)\right], \quad \phi_{V}>E_{z},
$$

where $\gamma$ is given in terms of the barrier height and width as

$$
\gamma=\frac{\sqrt{2} \pi^{2}}{h} \sqrt{\frac{m}{\phi_{0}}} d .
$$

In practical units, $\gamma$ is given by

$$
\gamma\left[(\mathrm{eV})^{-1}\right] \sim 0.805 \frac{d[\AA]}{\sqrt{\phi_{0}[\mathrm{eV}]}} .
$$

Instead of applying the WKB approximation we may consider a parabolic barrier which extends to $z= \pm \infty$ instead of being truncated at the metal surfaces as indicated by the dotted curves in Fig. 2. For this potential a simple and exact expression for the transmission, $T_{1 \mathrm{D}}^{\mathrm{P}}$, can be found $\mathrm{d}^{7,18,19}$

$$
T_{1 \mathrm{D}}^{\mathrm{P}}\left(E_{z}, V\right)=\frac{1}{1+\exp \left[\gamma\left(\phi_{V}-E_{z}\right)\right]} .
$$

Unlike the WKB result derived in Eq. (16), this formula is also valid for $E_{z}>\phi_{V}$. In the tunneling regime, it is instructive to rewrite Eq. (19) as ${ }^{20}$

$$
T_{1 \mathrm{D}}^{\mathrm{P}}=\frac{1}{1+1 / T_{1 \mathrm{D}}^{\mathrm{WKB}}}, \quad E_{Z}<\phi_{V} .
$$

In the opaque barrier limit $\left(T_{1 \mathrm{D}}^{\mathrm{WKB}} \ll 1\right)$, the WKB transmission is identical to the extended parabolic barrier transmission. For more transparent barriers, the WKB approximation gradually breaks down. In the extreme case $E_{z}=\phi_{V}$, where $T_{1 \mathrm{D}}^{\mathrm{WKB}}=1$, the parabolic result is $T_{1 \mathrm{D}}^{\mathrm{P}}=0.5$. By refining the WKB approximation, it can actually be shown that a better estimate of the transmission probability close to the top of an arbitrary barrier is given by Eq. (20). ${ }^{18,21,22}$

Although the expression for $T_{1 \mathrm{D}}^{\mathrm{P}}$ in Eq. (19) gives more reliable values for the transmission close to the top of the barrier, one might still question its validity at energies approaching the bottom the electrode potentials. Since the asymptotic wave functions for the extended parabolic barrier bare no resemblance to the plane waves found for the truncated parabolic barrier, this could give very different results. On the other hand, small values of $E_{z}$ correspond to opaque barriers, where the WKB approximation is known to hold fairly well. Since the WKB transmission depends only on the shape of the classically forbidden region (which is the same whether we truncate the parabola or not), the deviations may not be so large. To elucidate this point further, we have performed numerical ${ }^{23}$ calculations of the transmission $T_{1 \mathrm{D}}^{\mathrm{TP}}$ of the truncated parabolic barrier for different barrier parameters. Representative results are shown in Fig. 3 and compared with the analytical expressions for $T_{1 \mathrm{D}}^{\mathrm{P}}$ [Eq. (19)] and $T_{1 \mathrm{D}}^{\mathrm{WKB}}$ [Eq. (16)]. At zero bias voltage, the transmissions gradually saturate to one as the energy increases towards $\phi_{0}$. When a voltage of $2 \mathrm{~V}$ is applied, the barrier is lowered by $\approx 1 \mathrm{eV}$ [Eq. (15)], effectively shifting the curves to lower energies by $\approx 1 \mathrm{eV}$. As expected, the WKB approximation overestimates the transmission close to the top of the barrier. At lower energies, on the other hand, $T_{1 \mathrm{D}}^{\mathrm{TP}}>T_{1 \mathrm{D}}^{\mathrm{WKB}}$ as can be seen in the lower left panel. In general, $T_{1 \mathrm{D}}^{\mathrm{P}}$ closely follows $T_{1 \mathrm{D}}^{\mathrm{TP}}$.

Inserting Eq. (19) in Eq. (3) we get

$$
T_{3 \mathrm{D}}^{\mathrm{P}}(E, V)=\frac{1}{\gamma E} \ln \left\{1+\exp \left[-\gamma\left(\phi_{V}-E\right)\right]\right\},
$$

where we have omitted a constant term $\ln \left\{1+\exp \left[-\gamma \phi_{V}\right]\right\}$ since it is negligible in comparison to $\ln \left\{1+\exp \left[-\gamma\left(\phi_{V}\right.\right.\right.$ $-E)]\}$ for realistic values of the barrier parameters. In Fig. 4(a) we show the energy dependence of $T_{3 \mathrm{D}}^{\mathrm{P}}$ for the same parameters used previously in Fig. 3. For $E$ approaching $\phi_{0}$, the transmissions gradually roll of from the exponentially increasing WKB regime and saturate at a value below one.

Now we use Eq. (21) to calculate the mean transmission averaged over the active voltage window $\mathcal{T}^{\mathrm{P}}(V)$. Using the Sommerfeld expansion, we first calculate the zero temperature mean transmission by inserting Eq. (21) in Eq. (7) and solving the integral exactly. The result is

$$
\begin{aligned}
\mathcal{T}_{0}^{\mathrm{P}}(V)= & \frac{1}{\gamma^{2} E_{F} \mathrm{eV}}\left(\operatorname{Li}_{2}\left\{-\exp \left[-\gamma\left(\phi_{V}+\mathrm{eV}-E_{F}\right)\right]\right\}\right. \\
& \left.-\mathrm{Li}_{2}\left\{-\exp \left[-\gamma\left(\phi_{V}-E_{F}\right)\right]\right\}\right),
\end{aligned}
$$

where $\mathrm{Li}_{2}(z)$ is the dilogarithm function. ${ }^{24,25}$ The temperature correction $\Delta \mathcal{T}^{\mathrm{P}}$ can be found immediately by inserting Eq. (19) in Eq. (10) 


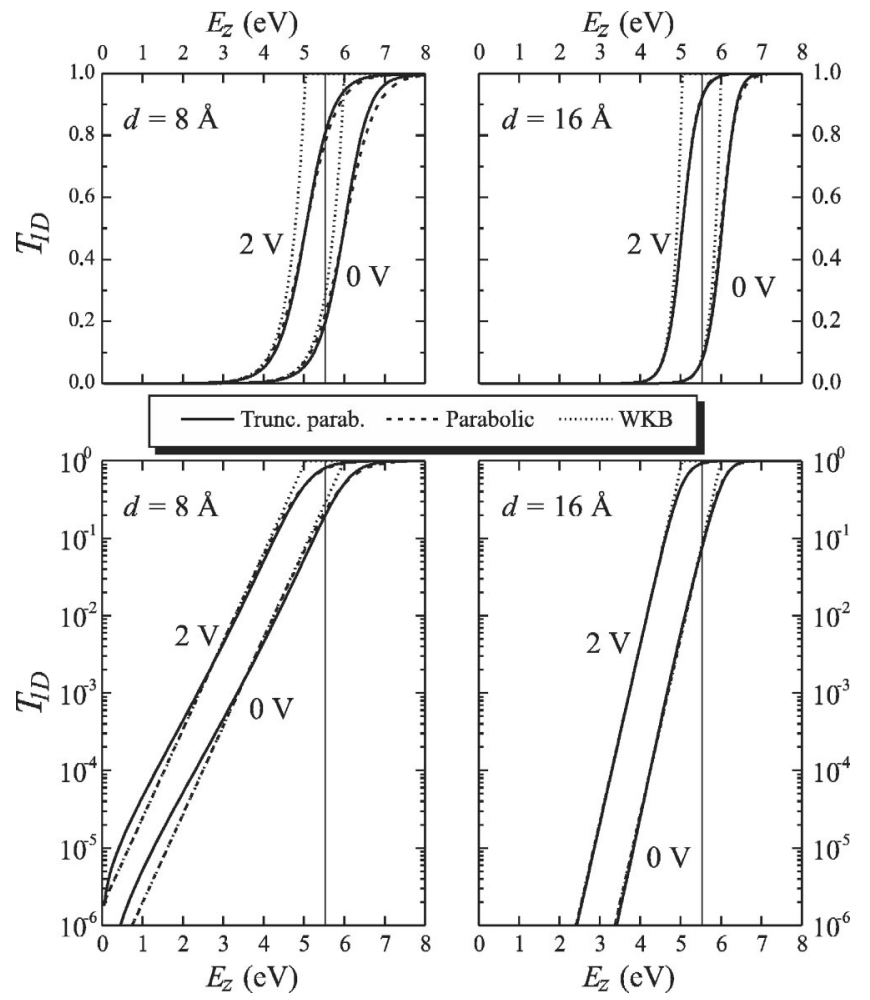

FIG. 3. The energy dependence of the 1D transmission through a truncated parabolic barrier with barrier height $\phi_{0}=6 \mathrm{eV}$ and electrodes with $E_{F}$ $=5.5 \mathrm{eV}, \lambda_{F}=5.2 \AA$, corresponding to gold (see Ref. 12). The transmissions are plotted for barrier thicknesses of $8 \AA$ (left panels) and $16 \AA$ (right panels) using a bias voltage of 0 and $2 \mathrm{~V}$ as indicated in the graphs. For each choice of barrier parameters, the transmission is calculated using three different methods: (i) the exact transmission $T_{1 \mathrm{D}}^{\mathrm{TP}}$ using a recursion method (see Ref. 23) (solid lines); (ii) the transmission $T_{1 \mathrm{D}}^{\mathrm{P}}$ through an extended parabolic barrier [Eq. (19)] (dashed lines); and (iii) the transmission $T_{1 \mathrm{D}}^{\mathrm{WKB}}$ calculated within the WKB approximation [Eq. (16)] (dotted lines). The transmissions are shown on both linear (upper panels) and logarithmic (lower panels) scales. The Fermi energy is indicated by a thin vertical line.

$$
\begin{aligned}
\Delta \mathcal{T}^{\mathrm{P}}(V)= & \frac{\pi^{2}}{6} \frac{\left(k_{B} T\right)^{2}}{E_{F} \mathrm{eV}}\left\{\frac{1}{1+\exp \left[\gamma\left(\phi_{V}-E_{F}\right)\right]}\right. \\
& \left.-\frac{1}{1+\exp \left[\gamma\left(\phi_{V}-E_{F}+\mathrm{eV}\right)\right]}\right\} .
\end{aligned}
$$

Equations (22) and (23) are the key results of this article and give the current-voltage characteristics, $I^{\mathrm{P}}(V)$ $=G_{S} \mathcal{T}^{\mathrm{P}}(V) V$, for the simple parabola model.

In Fig. 4(b) we show examples of the voltage dependence of $\mathcal{T}^{\mathrm{P}}$ for the same parameters used previously. When the voltage is increased, the transmission generally increases because of the barrier lowering which gives rise to nonlinearity. ${ }^{8}$ We also note that the thicker the barrier, the larger the nonlinearities. We have plotted the temperature correcting term $\Delta \mathcal{T}^{\mathrm{P}}$ for $T=300 \mathrm{~K}$ with thin lines. For the thin $8 \AA$ barrier, $\Delta \mathcal{T}^{P}$ is two to three orders of magnitude lower than $\mathcal{T}^{\mathrm{P}}$, and it can be neglected. However, when the thickness is increased, the temperature correction becomes increasingly important, and for the $24 \AA$ barrier (dasheddotted lines), it gives a 5\% contribution to the total transmission. This is because the energy dependence of $T_{3 \mathrm{D}}^{\mathrm{P}}$ increases with increasing thickness as seen from Fig. 4(a) and elec-
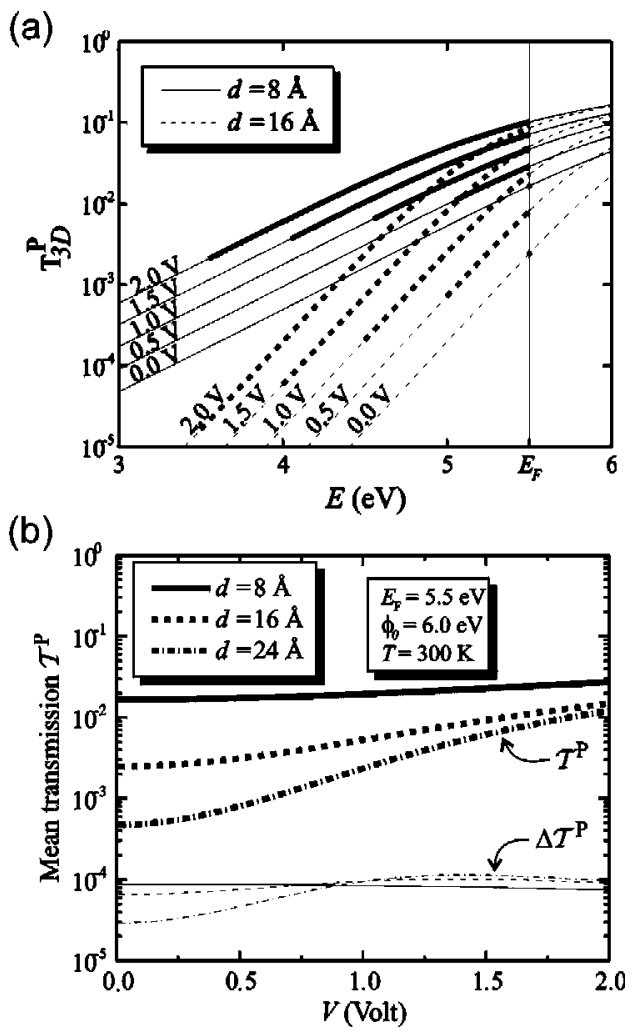

FIG. 4. (a) The energy dependence of the average transmission $T_{3 \mathrm{D}}^{\mathrm{P}}(E)$ for different voltages using the same parameters as in previous figures. The thick parts of the curves indicate the active energy window of the electrons, see Eq. (22). (b) Thick lines: voltage dependence of the mean transmission $\mathcal{T}^{\mathrm{P}}$ calculated using Eqs. (22) and (23) for different barrier thickness. Thin lines: the temperature correction to the mean transmission $\Delta \mathcal{T}^{\mathrm{P}}$ calculated using Eq. (23).

trons which are excited by an energy of the order $k_{B} T$ at the Fermi energy will have a significantly enhanced transmission.

For opaque barriers where the WKB approximation applies, the exponentials in Eq. (22) will be much less than one. Since $\mathrm{Li}_{2}(x) \sim x$ for $x \ll 1$, Eq. (22) reads

$$
\begin{aligned}
\mathcal{T}_{0}^{\mathrm{P}} \sim & \frac{\exp \left[-\gamma\left(\phi_{0}-E_{F}\right)\right]}{\gamma E_{F}} \\
& \times \exp \left[-\frac{\gamma}{16 \phi_{0}}(\mathrm{eV})^{2}\right] \frac{2 \sinh (\gamma \mathrm{eV} / 2)}{\gamma \mathrm{eV}} .
\end{aligned}
$$

In this limit we find the temperature correction,

$$
\frac{\Delta \mathcal{T}^{\mathrm{P}}}{\mathcal{T}^{\mathrm{P}}}=\frac{\pi^{2}}{6} \gamma^{2}\left(k_{B} T\right)^{2} \approx 7.9 \times 10^{-9} \frac{d[\AA]^{2} T[\mathrm{~K}]^{2}}{\phi_{0}[\mathrm{eV}]},
$$

which is only valid for sufficiently low temperature and small barrier thickness were the Sommerfeld expansion applies $\left(\gamma k_{B} T \ll 1\right)$. Formulas for the $I-V$ curve identical in form to Eqs. (24) and (25) can be shown to hold for arbitrary symmetric barrier within the WKB approximation. ${ }^{4}$

\section{SUMMARY}

We have derived a simple analytic result for the currentvoltage curve for tunneling of electrons through a simple parabolic barrier model. Our result for the current-voltage 
curve goes beyond the widely used WKB approximation by using a more accurate formula for the transmission. This makes the model well suited for calculating $I-V$ curves for thin barriers with small barrier heights. The only parameters in the model are the Fermi energy of the electrodes, the barrier height, and thickness. The model has previously been used to fit experimental nonlinear current-voltage curves. ${ }^{8}$ We have illustrated how temperature effects and nonlinearity in the $I-V$ curves become increasingly important as the thickness of the barrier is increased.

\section{ACKNOWLEDGMENTS}

The authors thank Dr. S. Kynde Nielsen and Professor F. Besenbacher, Professor I. Stensgaard, and Professor E. Lægsgaard for many fruitfull discussions. M.B. acknowledges support from the Danish Research Council (SNF).

${ }^{1}$ J. Frenkel, Phys. Rev. 36, 1604 (1930).

${ }^{2}$ A. Sommerfeld and H. Bethe, in Aufbau der Zusammenhängende Materie, edited by H. Geiger and K. Schell (Julius Springer-Verlag, Berlin, 1933), No. 24/2 in Handbuch der Physik, Chap. 3, pp. 443-454, 2nd ed.

${ }^{3}$ R. Holm and B. Kirschstein, Z. Tech. Phys. (Leipzig) 16, 488 (1935).

${ }^{4}$ R. Stratton, J. Phys. Chem. Solids 23, 1177 (1962).

${ }^{5}$ J. G. Simmons, J. Appl. Phys. 34, 1793 (1963).

${ }^{6}$ J. G. Simmons, J. Appl. Phys. 34, 2581 (1963).

${ }^{7}$ B. S. Jeffreys, in Quantum Theory, edited by D. R. Bates (Academic Press, New York, 1961), Vol. 1, Chap. 7, pp. 240-242.

${ }^{8}$ K. Hansen, S. K. Nielsen, M. Brandbyge, E. Lægsgaard, I. Stensgaard, and F. Besenbacher, Appl. Phys. Lett. 77, 708 (2000).

${ }^{9}$ M. Büttiker, Y. Imry, R. Landauer, and S. Pinhas, Phys. Rev. B 31, 6207 (1985).
${ }^{10}$ Y. Imry, in Directions in Condensed Matter Physics, edited by G. Grinstein and G. Mazenko (World Scientific, Singapore, 1986), pp. 101-163.

${ }^{11}$ Y. V. Sharvin, Zh. Eksp. Teor. Fiz. 48, 984 (1965) [Sov. Phys. JETP 21, 655 (1965)]

${ }^{12}$ N. W. Ashcroft and N. D. Mermin, Solid State Physics (Holt, Rinehart and Winston, New York, 1976).

${ }^{13}$ J. J. W. M. Rosink, M. A. Blauw, L. J. Geerligs, E. van der Drift, and S. Radelaar, Phys. Rev. B 62, 10459 (2000).

${ }^{14}$ R. E. Holmlin, R. Haag, M. L. Chabinyc, R. F. Ismagilov, A. E. Cohen, A. Terfort, M. A. Rampi, and G. M. Whitesides, J. Am. Chem. Soc. 123, 5075 (2001).

${ }^{15}$ R. Holm, J. Appl. Phys. 22, 569 (1951).

${ }^{16}$ J. G. Simmons, J. Appl. Phys. 35, 2474 (1964).

${ }^{17}$ S. Datta, W. Tian, S. Hong, R. Reifenberger, J. I. Henderson, and C. P. Kubiak, Phys. Rev. Lett. 79, 2530 (1997).

${ }^{18}$ E. C. Kemble, The Fundamental Principles of Quantum Mechanics with Elementary Applications (Dover, New York, 1937), pp. 109-112.

${ }^{19}$ L. D. Landau and E. M. Lifshitz, Quantum Mechanics. Non-Relativistic Theory, vol. 3 of Course of Theoretical Physics (Pergamon, London, 1965), 2nd revised ed., see $\$ 50$, problem 4.

${ }^{20}$ M. Brandbyge, J. Schiøtz, M. R. Sørensen, P. Stoltze, K. W. Jacobsen, J. K. Nørskov, L. Olesen, E. Lægsgaard, I. Stensgaard, and F. Besenbacher, Phys. Rev. B 52, 8499 (1995).

${ }^{21}$ S. C. Miller, Jr. and R. H. Good, Jr., Phys. Rev. 91, 174 (1953).

${ }^{22}$ L. I. Glazman, G. B. Lesovik, D. E. Khmel'nitskii, and R. I. Shekhter, Pis'ma Zh. Eksp. Teor. Fiz. 48, 218 (1988), [JETP Lett. 48, 238 (1988)].

${ }^{23}$ H. Q. Nguyen, P. H. Cutler, T. E. Feuchtwang, and N. Miskovsky, Surf. Sci. 160, 331 (1985).

${ }^{24}$ L. Lewin, Polylogarithms and Associated Functions (North Holland, New York, 1981)

${ }^{25}$ The dilogarithm is included in standard mathematical programs and libraries such as MATHEMATICA ${ }^{\mathrm{TM}}$ or the GNU scientific library www.gnu.org/ software/gsl. It can be approximated by simpler functions, see Y. Luke, The Special Functions and their Approximations (Academic Press, New York, 1969). 